\title{
Magnetosphere-ionosphere coupling at Jupiter: A parameter space study
}

\author{
L. C. Ray, ${ }^{1}$ R. E. Ergun, ${ }^{2,3}$ P. A. Delamere, ${ }^{2}$ and F. Bagenal ${ }^{2,3}$ \\ Received 1 June 2011; revised 2 November 2011; accepted 2 November 2011; published 7 January 2012.
}

[1] Jupiter's main auroral emission is a signature of the current system that transfers angular momentum from the planet to radially outward moving Iogenic plasma. Ray et al. (2010) developed a steady state model of this current system which self-consistently included the effects of a field-aligned potential, $\Phi_{\|}$, and an ionospheric conductance modified by precipitating electrons. The presented parameter space study extends their model to explore how variations in the auroral cavity density and temperature, magnetospheric mass loading rate, and background ionospheric Pedersen conductance affect the current system and resulting auroral emission. We show that while the solutions found by Ray et al. (2010) vary with changes in the system parameters, the gross general trends remain similar to the original solutions. We find that, for an outer constraint of $\mathrm{I}_{100}=86 \mathrm{MA}$, the high-latitude electron temperature and density have a lower limit of $\sim 1.5 \mathrm{keV}$ and an upper limit of $\sim 0.01 \mathrm{~cm}^{-3}$, respectively, in order for solutions to be consistent with observations of Jupiter's auroral emission. For increases in the radial mass transport rate and an outer constraint of $\Phi_{\|_{\text {Max }}}=75 \mathrm{kV}$ the auroral emission brightness increases.

Citation: Ray, L. C., R. E. Ergun, P. A. Delamere, and F. Bagenal (2012), Magnetosphere-ionosphere coupling at Jupiter: A parameter space study, J. Geophys. Res., 117, A01205, doi:10.1029/2011JA016899.

\section{Introduction}

[2] Jupiter displays three distinct types of aurora: satellite driven, rotation driven, and variable polar emission (see review by Clarke et al. [2004]). The satellite driven aurorae are the signature of the motions of Io, Europa, and Ganymede relative to Jupiter's magnetic field and magnetospheric equatorial plasma (see review by Saur et al. [2004]) and are the most equatorward emissions. The variable polar aurora, the highest latitude emission, maps to the outer magnetosphere and is likely driven by Jupiter's interaction with the solar wind [Cowley et al., 2003a; Delamere and Bagenal, 2010]. Between the satellite-driven and polar aurorae is the main auroral emission, a steady state structure fixed in magnetic longitude rotating with the planet.

[3] The main auroral emission is driven by the radial transport of Io-genic plasma through Jupiter's magnetosphere [Hill, 1979; Cowley and Bunce, 2001]. As plasma moves outwards via a centrifugally-driven interchange instability (see review by Krupp et al. [2004, and references therein]), one would expect it to slow down to conserve angular

\footnotetext{
${ }^{1}$ Space and Atmospheres Group, Department of Physics, Imperial College London, London, UK.

${ }^{2}$ Laboratory for Atmospheric and Space Physics, University of Colorado at Boulder, Boulder, Colorado, USA.

${ }^{3}$ Also at Department for Astrophysical and Planetary Sciences, University of Colorado at Boulder, Boulder, Colorado, USA.

Copyright 2012 by the American Geophysical Union. 0148-0227/12/2011JA016899
}

momentum. However, the subcorotation of the magnetospheric plasma relative to the planetary rotation drives currents that flow along the magnetic field from Jupiter to the magnetosphere and then radially outwards, providing an equatorial $\mathbf{J} \times \mathbf{B}$ force and transporting angular momentum from the planet to the plasma (see Figure 1). Due to the centrifugal confinement of the magnetospheric plasma to the equatorial plane, field-aligned potentials develop at highlatitudes to meet the magnetosphere's demand for current, accelerating electrons into Jupiter's atmosphere and creating Jupiter's main auroral emission. However, the currents cannot transport enough angular momentum to sustain rigid corotation throughout the entire magnetosphere and between $\sim 17$ and $\sim 20$ Jovian radii $\left(\mathrm{R}_{J}\right)$ the azimuthal flow begins to depart from corotation [McNutt et al., 1979; Krupp et al., 2001; Frank and Paterson, 2002]. The main auroral emission is associated with this current system.

[4] The characteristics of Jupiter's main auroral emission are summarized as follows: the emission occurs over a narrow extent in planetary latitude which maps to an equatorial distance of $\sim 20-30 \mathrm{R}_{J}$ [Clarke et al., 2004]; the emission is excited by $\sim 30-200 \mathrm{keV}$ electrons [Gustin et al., 2004] implying field-aligned potentials of a similar voltage, i.e. $30-200 \mathrm{kV}$; and the precipitating electron energy flux is $\sim 2-30 \mathrm{~mW} / \mathrm{m}^{2}$ [Gustin et al., 2004]. The physical parameters in the magnetosphere are similarly constrained through in situ measurements and observations. Delamere et al. [2005] determined that, after charge-exchange and fast neutral escape, $\sim 350-600 \mathrm{~kg} / \mathrm{s}$ of Io-genic plasma is available for radial transport. At $17 \mathrm{R}_{J}$ in the equatorial plane, the thermal 


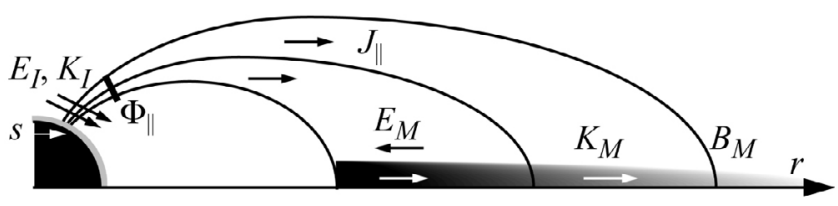

Figure 1. Diagram of coordinates and variables used in the model in the corotating frame. The model is $1 \mathrm{D}$ and all variables are a function of the radial position from the spin axis in the magnetosphere $(r)$. The corresponding distance from the spin axis in the ionosphere is $s$. The magnetic field model is assumed to be aligned with the spin-axis. The fieldaligned potential, marked by the bar, is expected to develop close to Jupiter.

core of electrons has a temperature of $\sim 20-50 \mathrm{eV}$ and a density of $\sim 2-6 \mathrm{~cm}^{-3}$ while the hot electron population has an energy of $\sim 2.5 \mathrm{keV}$ and a density of $0.01 \mathrm{~cm}^{-3}$ [Scudder et al., 1981]. Reconciling the theory of angular momentum transport with observations of Jupiter's main auroral emission and in situ measurements of the magnetosphere has been the topic of many analyses [Hill, 1979; Pontius, 1997; Cowley and Bunce, 2001; Cowley et al., 2003b; Nichols and Cowley, 2004, 2005; Ray et al., 2010] which we will briefly review below.

[5] Hill [1979] describes the transfer of angular momentum from Jupiter to its magnetospheric plasma by equating the torque of the radially moving magnetospheric plasma with that from ion-neutral collisions in Jupiter's atmosphere. The theoretical description uses a dipole magnetic field and constant ionospheric Pedersen conductance. Subsequent theoretical models build upon this analysis by modifying the Pedersen conductance to account for the sub-corotation of the neutral atmosphere [Huang and Hill, 1989] and applying a stretched equatorial magnetic field configuration [Pontius, 1997].

[6] Cowley and Bunce [2001] present the first numerical model of the current system, comparing the resulting fieldaligned current densities and structures to auroral observations. Further studies investigate the dependence of the angular momentum transport on the magnetic field structure [Cowley et al., 2003b] and on modifications of the ionospheric Pedersen conductance, $\Sigma_{P}$, by precipitating auroral electrons [Nichols and Cowley, 2004]. Nichols and Cowley [2005] included the effect of field-aligned potentials on the transfer of angular momentum between Jupiter and the magnetosphere for a system with a constant ionospheric Pedersen conductance. The latter two studies determine the energy flux of the precipitating electrons and the field-aligned potentials using the linear approximation of the Knight [1973] currentvoltage relation as derived by Lyons [1980].

[7] The Knight [1973] current-voltage relation assumes a monotonic potential structure between the plasma sheet and ionosphere. The electron temperature and density are defined by the population in the plasma sheet and the motion of the electrons along the flux tube is dictated by mirror forces. The Knight [1973] current-voltage relation can be approximated linearly in the regime where $1 \ll e \Phi_{\|} / k T_{e} \ll R_{x}$ where $R_{x}$ is the mirror ratio between the equator and the top of the acceleration region (i.e. when the electron potential energy is greater than the electron thermal energy, but not to the extent that the electron distribution function is appreciably depleted) [Lyons, 1980].

[8] Ray et al. [2009] show that the linear approximation to the Knight [1973] current-voltage relation [Lyons, 1980] is not applicable in rapidly rotating systems with an internal plasma source. The rapid rotation centrifugally confines heavy ions to the equatorial plane which results in an ambipolar electric field that restricts the mobility of the magnetospheric electrons. This confinement, along with the gravitational confinement of the ionospheric plasma population, leads to a low plasma density at high latitudes. A field-aligned potential drop develops at this location and it is the plasma population and magnetic field strength at this location that dictate the nature of the current-voltage relation. In Jupiter's middle magnetosphere, the hot electron population has an energy of $\sim 2.5 \mathrm{keV}$, and the mirror ratio from the top of the acceleration region, located at $\sim 2-3 \mathrm{R}_{J}$ Jovicentric, to the planet is $R_{x} \sim 16$. Assuming the fieldaligned potentials inferred from observations of $30-200 \mathrm{kV}$, the condition for the linear approximation of the Knight [1973] relation fails. The resulting current-voltage relation, dubbed the "high-latitude current choke" by Ray et al. [2009], is the fully exponential Knight [1973] currentvoltage relation applied at the top of the acceleration region rather than the magnetospheric equatorial plane. At $6 \mathrm{R}_{J}$, applying the current-voltage relation at the magnetosphere rather than at high-latitudes results in an over-estimate of the field-aligned current densities by two orders of magnitude [Ray et al., 2009].

[9] The presence of field-aligned potentials allows for differential rotation between the magnetosphere and ionosphere and alters the perpendicular electric field mapping between the two regions. The field-aligned potentials can significantly affect angular momentum transport if the potential drops are comparable to the rotational potential [Mauk et al., 2002]. Ray et al. [2010] show that for the Jovian system, the field-aligned potentials are a significant fraction of the rotational potential and therefore need to be selfconsistently treated when describing the current system associated with angular momentum transport.

[10] Ray et al. [2010] describe a 1-D steady state model of the magnetosphere-ionosphere coupling system at Jupiter. The model develops the electric fields, currents, ionospheric Pedersen conductances, and field-aligned potentials which result from the radial transport of Io-genic plasma outward through the Jovian system and subsequent transfer of angular momentum from Jupiter to the equatorial plasma. The model uses the 'high-latitude current choke' current voltage relation and has several input parameters; the radial mass transport rate, $\dot{M}$; electron density and temperature at high-latitude, $n_{x}$ and $T_{x}$, respectively; the location of the acceleration region at high-latitudes, $R_{x}$; background Pedersen conductance at the ionosphere, $\Sigma_{P_{0}}$; and the efficiency of the modification of the Pedersen conductance, $\epsilon$. The input parameters are consistent with measurements of the Jovian system and past analyses as described above.

[11] Only the upward current system is described by the Ray et al. [2010] model and as such the model does not include the entire circuit. Sources and sinks of energy are not fully balanced and the system is under-constrained, resulting in a set of solutions. Therefore their model requires an outer 
Table 1. Symbols and Parameters Used in the Model

\begin{tabular}{|c|c|c|c|}
\hline Symbol & Description & Type & Units \\
\hline$\alpha(r)$ & $\begin{array}{l}\text { Magnetosphere-ionosphere radial } \\
\text { field mapping }\end{array}$ & Prescribed & - \\
\hline $\mathrm{B}_{M}(\mathrm{r})$ & Magnetic field in magnetosphere & Prescribed & $\mathrm{T}$ \\
\hline $\mathrm{E}_{I}(\mathrm{r})$ & $\begin{array}{l}\text { Ionospheric electric field in corotating } \\
\text { frame }\end{array}$ & Variable & $\mathrm{V} \mathrm{m}^{-}$ \\
\hline $\mathrm{E}_{M}(\mathrm{r})$ & $\begin{array}{l}\text { Magnetospheric motional electric } \\
\text { field in corotating frame }\end{array}$ & Variable & $\mathrm{V} \mathrm{m}^{-1}$ \\
\hline $\mathrm{E}_{I}^{*}(\mathrm{r})$ & $\begin{array}{l}\text { Ionospheric electric field mapped to } \\
\text { the magnetosphere }\end{array}$ & Variable & $\mathrm{V} \mathrm{m}^{-1}$ \\
\hline$\Phi_{\|}(\mathrm{r})$ & $\begin{array}{l}\text { Field-aligned potential between } \\
\text { ionosphere and magnetosphere }\end{array}$ & Variable & V \\
\hline $\mathrm{J}_{\|}^{M}(\mathrm{r})$ & $\begin{array}{l}\text { Field-aligned current density at the } \\
\text { magnetosphere }\end{array}$ & Variable & $\mathrm{A} \mathrm{m}^{-2}$ \\
\hline $\mathrm{J}_{\|}^{I}(\mathrm{r})$ & $\begin{array}{l}\text { Field-aligned current density at the } \\
\text { ionosphere }\end{array}$ & Variable & $\mathrm{A} \mathrm{m}^{-2}$ \\
\hline $\mathrm{K}_{I}(\mathrm{r})$ & $\begin{array}{l}\text { Height-integrated current } \\
\text { (ionosphere) }\end{array}$ & Variable & $\mathrm{A} \mathrm{m}^{-}$ \\
\hline $\mathrm{K}_{M}(\mathrm{r})$ & $\begin{array}{l}\text { Height-integrated current } \\
\text { (magnetosphere) }\end{array}$ & Variable & $\mathrm{A} \mathrm{m}^{-1}$ \\
\hline$\dot{M}$ & $\begin{array}{l}\text { Radial transport rate of plasma mass } \\
\text { from Io torus }\end{array}$ & Constant & $\mathrm{kg} \mathrm{s}^{-1}$ \\
\hline $\mathrm{r}$ & $\begin{array}{l}\text { Equatorial radial position in } \\
\text { magnetosphere }\end{array}$ & Ordinant & $\mathrm{m}$ \\
\hline $\mathrm{R}_{M}(\mathrm{r})$ & Magnetic mirror ratio & Prescribed & - \\
\hline$\Sigma_{P}(\mathrm{r})$ & $\begin{array}{l}\text { Height-integrated Pedersen } \\
\text { conductivity }\end{array}$ & Variable & $\Omega^{-1}$ \\
\hline$\Omega(\mathrm{r})$ & Local rotation rate & Variable & $s^{-1}$ \\
\hline$\Omega_{J}$ & Jupiter's rotation rate & Constant & $\mathrm{s}^{-1}$ \\
\hline$\omega(\mathrm{r})$ & Deviation from corotation: $\Omega(\mathrm{r})-\Omega_{J}$ & Variable & $\mathrm{s}^{-1}$ \\
\hline
\end{tabular}

constraint to select a solution. Ray et al. [2010] choose either the total radial current at $100 \mathrm{R}_{J}, \mathrm{I}_{100}$, or the maximum field-aligned potential, $\Phi_{\|_{\operatorname{Max}}}$, as the outer constraint for the upward current system.

[12] In this paper we extend the Ray et al. [2010] analysis to explore how variations in the density of the current carrying electrons, $n_{x}$, the temperature of the high-latitude electrons, $T_{x}$, the radial mass transport rate, $\dot{M}$, and the baseline Pedersen conductance at the ionosphere, $\Sigma_{P_{0}}$, affect the angular momentum transfer from Jupiter to its equatorial plasma and the subsequent fields, currents systems, and auroral properties. We choose the same outer constraints as the Ray et al. [2010] analysis, either $\mathrm{I}_{100}=86 \mathrm{MA}$ or $\Phi_{\|_{M a x}}=$ $75 \mathrm{kV}$. The selection of $\mathrm{I}_{100}=86 \mathrm{MA}$ is derived from the Khurana [2001] analysis of Galileo magnetometer data while $\Phi_{\|_{\text {Max }}}=75 \mathrm{kV}$ is consistent with the range of precipitating electron energies derived by Gustin et al. [2004].

\section{Model Overview}

[13] We start with the same set of equations used in previous models [Pontius and Hill, 1982; Nichols and Cowley, 2004]. All symbols are described in Table 1, and Figure 1 shows the geometry. All variables are a function of radial distance in the equatorial plane as our model is 1D and assumes that the magnetic field is aligned with the spin axis. The model also assumes that Jupiter's ionosphere and plasma sheet are infinitely thin and cylindrically symmetric. The equations and solution method are briefly summarized below and the reader is referred to Ray et al. [2010] for a more detailed description.

[14] Calculations are made in Jupiter's corotating reference frame where the electric field represents deviation from rigid corotation. Following the analyses of Hill [1979], Pontius [1997], and Nichols and Cowley [2004, 2005], we begin with torque balance in the equatorial plane between the outward moving plasma and the $\mathbf{J} \times \mathbf{B}$ force from the subsequent currents:

$$
\dot{M} \frac{d}{d r}\left(r^{2} \Omega(r)\right)=2 \pi r^{2} K_{M}(r) B_{M}(r)
$$

The radial mass transport rate, $\dot{M}$, is assumed to be constant through the system and we use the CAN-KK magnetic field model to determine the north-south component of the equatorial field, $B_{M}(r)$ [Nichols and Cowley, 2004, 2005]. The total angular velocity of the plasma is

$$
\Omega(r)=\Omega_{J}+\omega(r)
$$

where $\omega(r)$ is the deviation in the angular velocity from corotation, $\Omega_{J}$ is the angular velocity of Jupiter, and $\Omega(r)$ is the total angular velocity of the magnetospheric plasma. The magnetospheric electric field in the corotating frame is given by:

$$
E_{M}(r)=\omega(r) r B_{M}(r)
$$

which initially maps directly to the ionospheric electric field $\left(E_{I}\right)$ in steady state $(\vec{\nabla} \times \vec{E}=0)$

$$
E_{I}(r)=\alpha(r) E_{M}(r)
$$

where the mapping function, $\alpha(r)$, is defined through conservation of magnetic flux and ranges from $\sim 20$ at $5 \mathrm{R}_{J}$ to $\sim 11000$ at $100 \mathrm{R}_{J}$. The height integrated ionospheric current density, $K_{I}$, is determined using Ohm's law for a given height-integrated Pedersen conductivity, $\Sigma_{P}$, yielding

$$
K_{I}(r)=\Sigma_{P} E_{I}(r)
$$

which defines the magnetospheric height-integrated current density

$$
K_{M}(r)=-2 K_{I}(r) \frac{s(r)}{r}
$$

We assume that both hemispheres respond identically accounting for the factor of two.

[15] Using current continuity, the field-aligned current densities at the magnetosphere and ionosphere are

$$
J_{\|}^{M}(r)=\frac{1}{r} \frac{d}{d r}\left(\frac{r K_{M}(r)}{2}\right)
$$

and

$$
J_{\|}^{I}(r)=R_{M}(r) J_{\|}^{M}(r)
$$

where $R_{M}(r)$ is the magnetic mirror ratio.

[16] Equations (1)-(6) ignore $\Phi_{\|}$and hold $\Sigma_{P}$ constant (equation (5)). To self-consistently include field-aligned potentials in the mapping of the ionospheric and magnetospheric electric fields, equation (4) $(\vec{\nabla} \times \vec{E}=0)$, is modified as

$$
E_{I}(r)=\alpha(r)\left(E_{M}(r)-\frac{d \Phi_{\|}(r)}{d r}\right)
$$




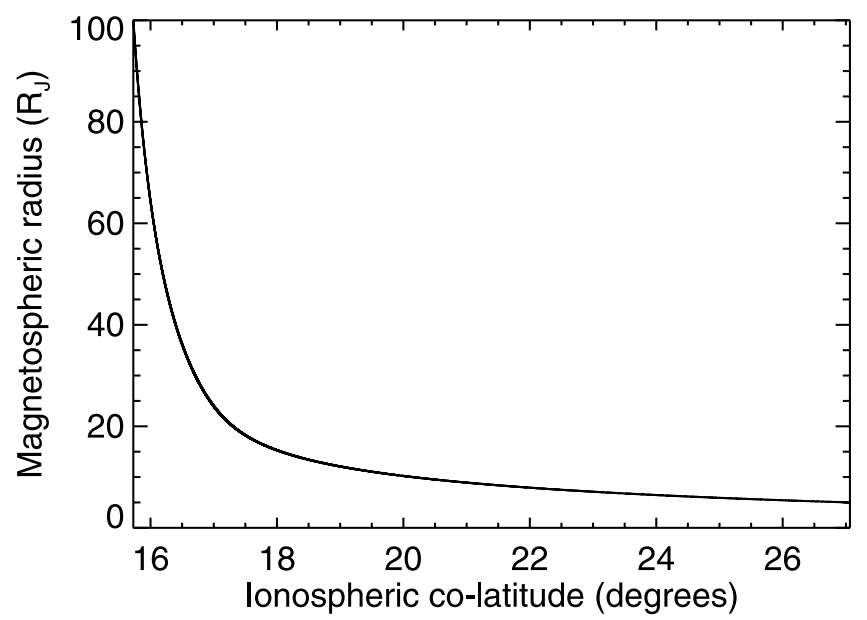

Figure 2. Mapping relationship between ionospheric colatitude and magnetospheric radius.

for a steady state, upward current system. The term $d \Phi_{\|} / d r$ represents the radial derivative of the field-aligned potential between the ionosphere and magnetosphere (i.e. the perpendicular derivative of the potential). The derivative is evaluated in the equatorial plane. We define

$$
E_{I}^{*}(r)=E_{I}(r) / \alpha(r)
$$

to represent the ionospheric electric field mapped to the magnetosphere. Figure 2 shows the ionospheric co-latitudes and corresponding mapped equatorial distances.

[17] Following Ray et al. [2009] we use the "high-latitude current choke" current voltage relation

$$
J_{\|}^{I}(r)=j_{x}+j_{x}\left(R_{x}-1\right)\left(1-e^{-\left(\frac{e \Phi}{T_{x}\left(R_{x}-1\right)}\right)}\right)
$$

where $J_{\|}^{I}$ is the field-aligned current density at the ionosphere, $j_{x}=e n_{x} \sqrt{T_{x} /\left(2 \pi m_{e}\right)}$ is the electron thermal current density, $R_{x}$ is the magnetic mirror ratio at the top of the acceleration region (located 2-3 $\mathrm{R}_{J}$ Jovicentric), $T_{x}$ and $n_{x}$ are the electron energy (in $\mathrm{eV}$ ) and density, $m_{e}$ is the electron mass, and $e$ is the fundamental charge. The subscript $(x)$ indicates that the quantities are chosen to reflect those at the top of the acceleration region, which is located where the sum of the gravitational and centrifugal potentials along the flux tube is a minimum [Ray et al., 2009]. The value of $j_{x}$ at this location is hereafter referred to as the critical current density, $J_{\text {crit }}$. Equation (11) is valid only for $J_{\|}^{I} \geq J_{\text {crit }}$, otherwise $\Phi_{\|}=0$ and $J_{\|}^{I}$ is calculated through equation (8).

[18] Where $J_{\|}^{I} \geq J_{\text {crit }}$, the Pedersen conductance to varies with incident energy flux $\left(E F=J_{\|} \Phi_{\|}\right)$and precipitating electron energy such that equation (5) becomes:

$$
K_{I}(r)=\Sigma_{P}\left(\Phi_{\|}, E F\right) E_{I}(r)
$$

The modified Pedersen conductance is

$$
\Sigma_{P}\left(\Phi_{\|}, E F\right)=\Sigma_{P_{0}}+\epsilon \frac{\Sigma_{P_{\Phi_{\|}}}\left(\Phi_{\|}\right) \Sigma_{P_{E F}}(E F)}{\Sigma_{P_{E F}}\left(10 m W / m^{2}\right)}
$$

where $\Sigma_{P_{0}}$ is the height-integrated Pedersen conductance in non-auroral regions, $\Sigma_{P_{\Phi}}\left(\Phi_{\|}\right)$is the contribution to the Pedersen conductance due to electron precipitation energy,

$$
\Sigma_{P_{\Phi_{\|}}}\left(\Phi_{\|}\right)=\left\{\begin{array}{cc}
A_{1} \Phi_{\|}+A_{2} \Phi_{\|}^{2}+A_{3} \Phi_{\|}^{3} & : \quad \Phi_{\|} \leq 93 \mathrm{keV} \\
0.76326113 \frac{e^{-\left(\Phi_{\|} / 1 \mathrm{keV}\right)^{1 / 4}}}{e^{-(93 \mathrm{keV} / 1 \mathrm{keV})^{1 / 4}}} \quad: \quad & \Phi_{\|}>93 \mathrm{keV}
\end{array}\right.
$$

where $A_{1}=4.58164 \times 10^{-6}, A_{2}=2.49649 \times 10^{-10}$ and $A_{3}=$ $-2.26522 \times 10^{-15}, \Sigma_{P_{E F}}(E F)$ is the contribution due to precipitating electron energy flux [Millward et al., 2002],

$$
\Sigma_{P_{E F}}(E F)=\left(E F^{\alpha_{P}} 10^{\gamma_{P}}\right) 10^{\beta_{P}\left(\log _{10} E F\right)^{2}}
$$

where $\alpha_{P}=0.437, \beta_{P}=0.089$ and $\gamma_{P}=-1.438$, and $\epsilon$ is the efficiency of the Pedersen conductance enhancement. The full details of the above Pedersen conductance formulation are given by Ray et al. [2010]. As in previous analyses [e.g., Hill, 1979] we set $\Sigma_{P_{0}}=0.1$ mho and $\epsilon=1$ unless otherwise stated.

[19] Equations (1)-(3) and (5)-(11) represent a closed set that includes field-aligned potentials generated by fieldaligned currents. These equations can be rewritten as two coupled differential equations, one that is second order in $\Phi_{\|}$ and first order in $\omega$ and one that is first order in $\Phi_{\|}$and $\omega$.

[20] The coupled set of equations can be numerically solved by setting three boundary conditions: (1) the initial deviation from corotation, $\omega_{0} ;(2)$ the initial field-aligned potential, $\Phi_{\|_{0}}$; and (3) the initial radial gradient of the fieldaligned potential, $\left(\frac{d \Phi_{\|}}{d r}\right)_{0}$.

[21] Initially, the solution follows from the $\Phi_{\|}=0$ approximation (equations (1)-(6)) up to the location where $J_{\|}^{I}$ first exceeds $J_{\text {crit }}$, hereafter called $r_{\text {crit }}$. We use the deviation from corotation at $r_{c r i t}$ to define $\omega_{0}$ and $\Phi_{\|_{0}}$ is calculated from $J_{\|}^{I}$ using equation (11). The third boundary condition, $\left(\frac{d \Phi_{\|}}{d r}\right)_{0}$, is estimated by carrying out the $\Phi_{\|}=0$ approximation one step past $r_{c r i t}$, and calculating the resulting $\left(\frac{d \Phi_{\|}}{d r}\right)_{0}$. Past $r_{\text {crit }}$, the solution is determined by equations (1)-(3) and (5)-(13) to self-consistently include field-aligned potentials and a variable Pedersen conductance. The resulting solution is then compared to an outer constraint, either the total radial current at $100 \mathrm{R}_{J}, \mathrm{I}_{100}$, or the maximum field-aligned potential, $\Phi_{\|_{M a x}}$ and $\left(\frac{d \Phi_{\|}}{d r}\right)_{0}$ is accordingly adjusted such that the outer constraint is met.

\section{Baseline Solution}

[22] In this analysis we will compare our results to the solution presented by Ray et al. [2010, section 4.2], hereafter referred to as the baseline solution, with an outer constraint of $\mathrm{I}_{100}=86 \mathrm{MA}, \dot{M}=1000 \mathrm{~kg} / \mathrm{s}, \Sigma_{P_{0}}=0.1 \mathrm{mho}, T_{x}=$ $2.5 \mathrm{keV}, n_{x}=0.01 \mathrm{~cm}^{-3}$, and $R_{x}=16$. This solution is shown in Figure 3 and summarized below.

[23] The critical radius for the parameters above is $r_{c r i t}=$ $15.1 \mathrm{R}_{J}$ at which location we set $\left(\frac{d \Phi_{\|}}{d r}\right)_{0}=1.3 \times 10^{-5}$. The 


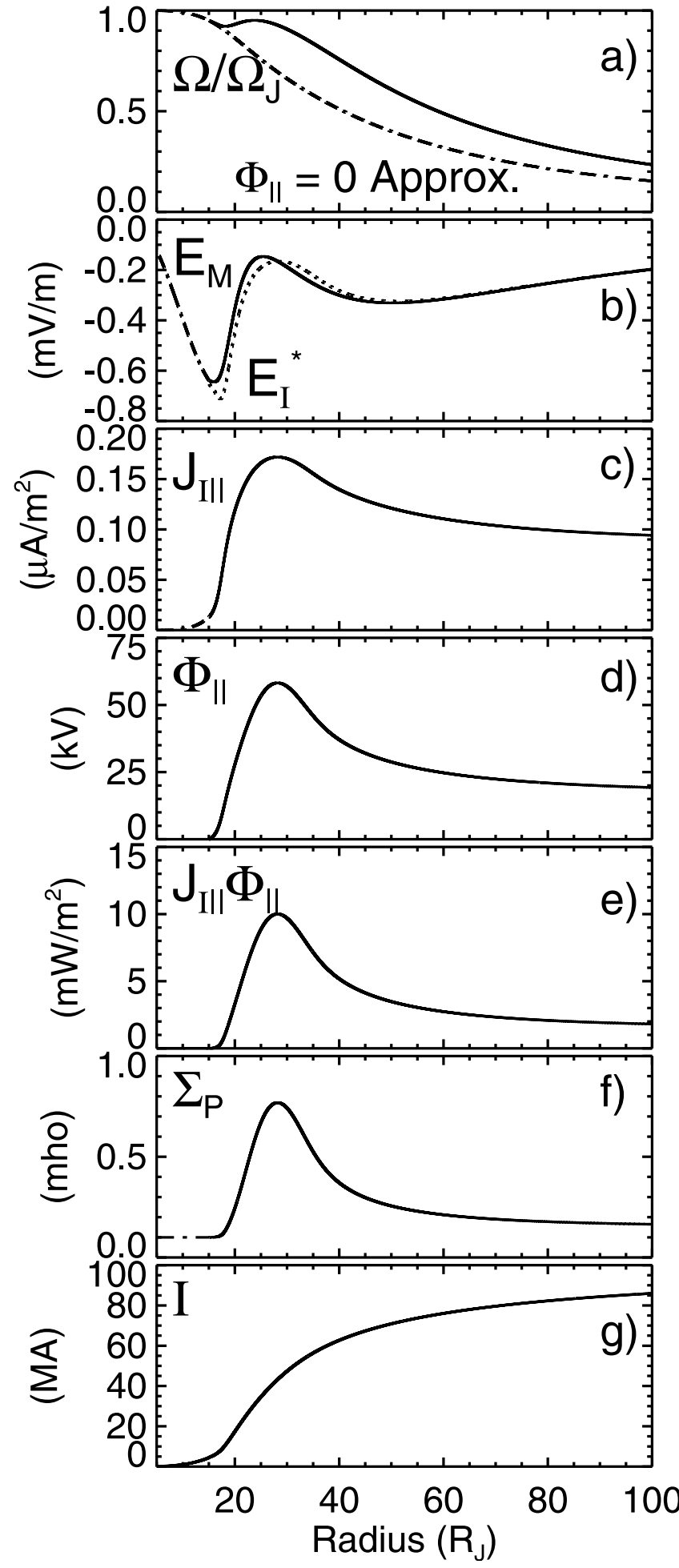

Figure 3. Model results for our baseline solution. (a) Rotation profile of plasma in the magnetosphere. The solution for the $\Phi_{\|}=0$ approximation profile is shown for reference (dot-dash-dash line). (b-g) The magnetospheric (dashed line) and mapped ionospheric (solid line) electric fields, the current density in the ionosphere, the field-aligned potential, the incident energy flux at the ionosphere, the height-integrated Pedersen conductivity, and the total radial current. field-aligned potentials boost the electron distribution into the loss cone increasing the field-aligned current density and accelerating electrons into the ionosphere; both effects of which modify the ionospheric Pedersen conductance. As per Ohm's law (equation (12)) if there is a sharp increase in $\Sigma_{P}$ then either the magnitude of the ionospheric electric field must decrease, the magnitude of the ionospheric heightintegrated current density must grow, or both $\left|E_{I}^{*}\right|$ and $K_{I}$ must vary. The field-aligned potentials modify the Pedersen conductance and $d \Phi_{\|} / d r$ affects the ionospheric electric field, therefore both the magnitude of the ionospheric electric field decreases as $\Sigma_{P}$ increases and the magnitude of $K_{I}$ grows. The magnitude of the mapped ionospheric electric field $\left(\left|E_{I}^{*}\right|\right)$ is larger than that of the magnetospheric electric field where $d \Phi_{\|} / d r$ is positive, and smaller than that of the magnetospheric electric field $\left(\left|E_{M}\right|\right)$ where $d \Phi_{\|} / d r$ is negative.

[24] The $\mathbf{I} \times \mathbf{B}$ force in the equatorial plane increases with the field-aligned current density. The angular velocity of the plasma stays near corotation until $\sim 30 \mathrm{R}_{J}$. Past $\sim 30 \mathrm{R}_{J}$ the $\mathbf{I} \times \mathbf{B}$ force is too weak due to the decreasing magnetic field strength to keep the plasma near corotation and the plasma angular velocity declines following a profile similar to that of the $\Phi_{\|}=0$ approximation.

[25] The above parameters result in a main auroral emission that maps to $28 \mathrm{R}_{J}$ with a half-width of $\sim 10 \mathrm{R}_{J}$. The maximum energy flux and electron precipitation energy are $\sim 10 \mathrm{~mW} / \mathrm{m}^{2}$ and $\sim 60 \mathrm{keV}$, respectively, and are consistent with the energy fluxes and electron precipitation energies derived from HST observations.

\section{Parameter Space Study}

[26] Each section below explores how the solutions vary with changes in the input parameters. For easy comparison, one parameter is varied while the other inputs are held fixed to the values used for our baseline solution: $\dot{M}=1000 \mathrm{~kg} / \mathrm{s}$, $R_{x}=16, \Sigma_{P_{0}}=0.1 \mathrm{mho}, T_{x}=2.5 \mathrm{keV}$, and $n_{x}=0.01 \mathrm{~cm}^{-3}$. We use an outer constraint of $\mathrm{I}_{100}=86 \mathrm{MA}$ for the parameter space studies with two exceptions. The study that varies the high-latitude electron density, $n_{x}$, uses an outer constraint of $\Phi_{\|_{\operatorname{Max}}}=75 \mathrm{kV}$ as there are no solutions with an $\mathrm{I}_{100}=86 \mathrm{MA}$ for large $n_{x}$, and we show solutions for both $\Phi_{\|_{\operatorname{Max}}}=75 \mathrm{kV}$ and $\mathrm{I}_{100}=86 \mathrm{MA}$ for the $\dot{M}$ case. Each figure displays the variation in solutions using the same format. From top to bottom, panels show the normalized $\mathbf{I} \times \mathbf{B}$ force, fieldaligned current density, angular velocity of the magnetospheric plasma normalized to corotation where the solid black line indicates full corotation, energy flux incident on the ionosphere, and radial current (outer constraint of $\Phi_{\|_{M a x}}=$ $75 \mathrm{kV}$ ) or Pedersen conductance (outer constraint of $\mathrm{I}_{100}=$ $86 \mathrm{MA})$. The dot-dash-dashed lines display the solution to the $\Phi_{\|}=0$ approximation for comparison.

[27] The corresponding tables list the maximum energy flux incident at the ionosphere, approximate width of the auroral emission at the planet, the location of the auroral emission when mapped to the magnetospheric equatorial plane, maximum field-aligned potential or total current at $100 \mathrm{R}_{J}$ depending on the outer constraint, and the maximum plasma angular velocity after $r_{c r i t}$. It should be noted that the auroral widths quoted are full-width half maximums. While the energy flux profiles are not perfect gaussians, this gives a 

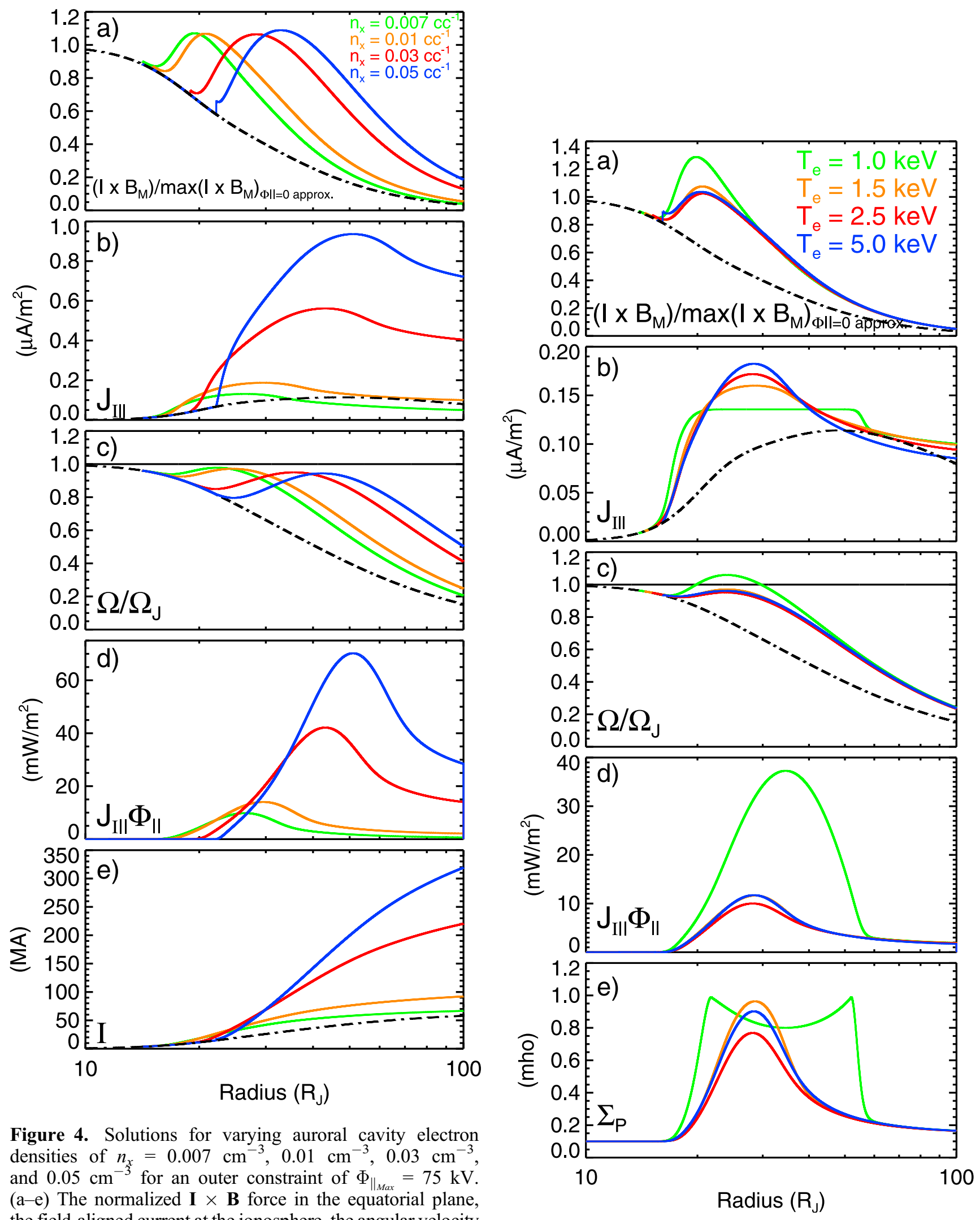

Figure 4. Solutions for varying auroral cavity electron densities of $n_{x}=0.007 \mathrm{~cm}^{-3}, 0.01 \mathrm{~cm}^{-3}, 0.03 \mathrm{~cm}^{-3}$, and $0.05 \mathrm{~cm}^{-3}$ for an outer constraint of $\Phi_{\|_{M a x}}=75 \mathrm{kV}$. (a-e) The normalized $\mathbf{I} \times \mathbf{B}$ force in the equatorial plane, the field-aligned current at the ionosphere, the angular velocity profile of the magnetospheric plasma, the energy flux incident on the ionosphere, and the radial current. The dot-dash-dash line is the solution to the $\Phi_{\|}=0$ approximation for comparison. The $\mathbf{I} \times \mathbf{B}$ forces are normalized to the maximum $\mathbf{I} \times \mathbf{B}$ force from the $\Phi_{\|}=0$ approximation.

Figure 5. (a-e) Solutions for varying electron temperatures of $T_{x}=1.0 \mathrm{keV}, 1.5 \mathrm{keV}, 2.5 \mathrm{keV}$, and $5.0 \mathrm{keV}$ for an outer constraint of $\mathrm{I}_{100}=86$ MA. Format is similar to Figure 4 except that Figure 5e displays the height-integrated Pedersen conductivity. 

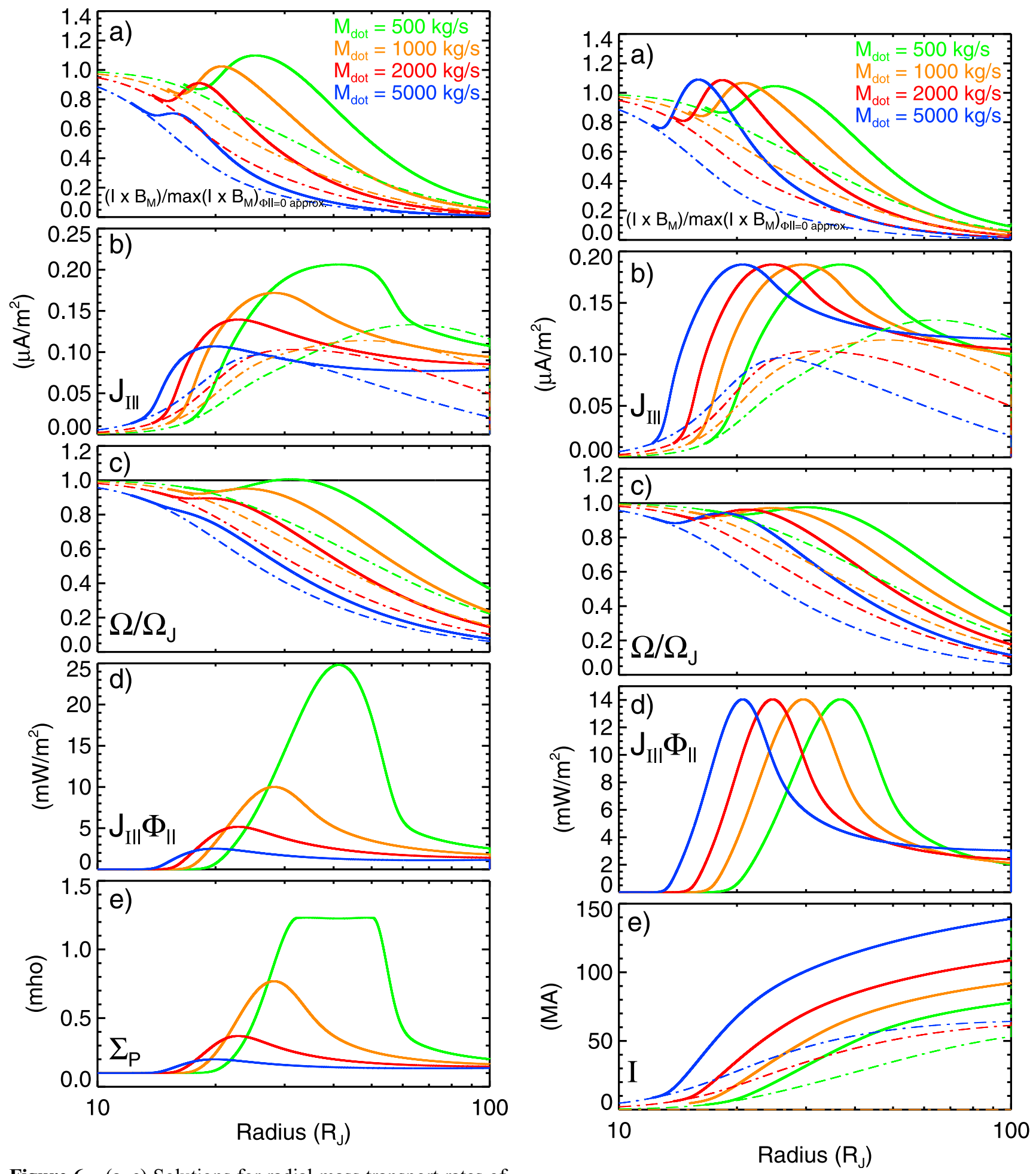

Figure 6. (a-e) Solutions for radial mass transport rates of $\dot{M}=500 \mathrm{~kg} / \mathrm{s}, 1000 \mathrm{~kg} / \mathrm{s}, 2000 \mathrm{~kg} / \mathrm{s}$, and $5000 \mathrm{~kg} / \mathrm{s}$ for an outer constraint of $\mathrm{I}_{100}=86 \mathrm{MA}$. Format is similar to Figure 4 except that Figure 6e displays the height-integrated Pedersen conductivity.

Figure 7. (a-e) Solutions for radial mass transport rates of $\dot{M}=500 \mathrm{~kg} / \mathrm{s}, 1000 \mathrm{~kg} / \mathrm{s}, 2000 \mathrm{~kg} / \mathrm{s}$, and $5000 \mathrm{~kg} / \mathrm{s}$ with an outer constraint of $\Phi_{\|_{M a x}}=75 \mathrm{kV}$. Format is similar to Figure 4. 

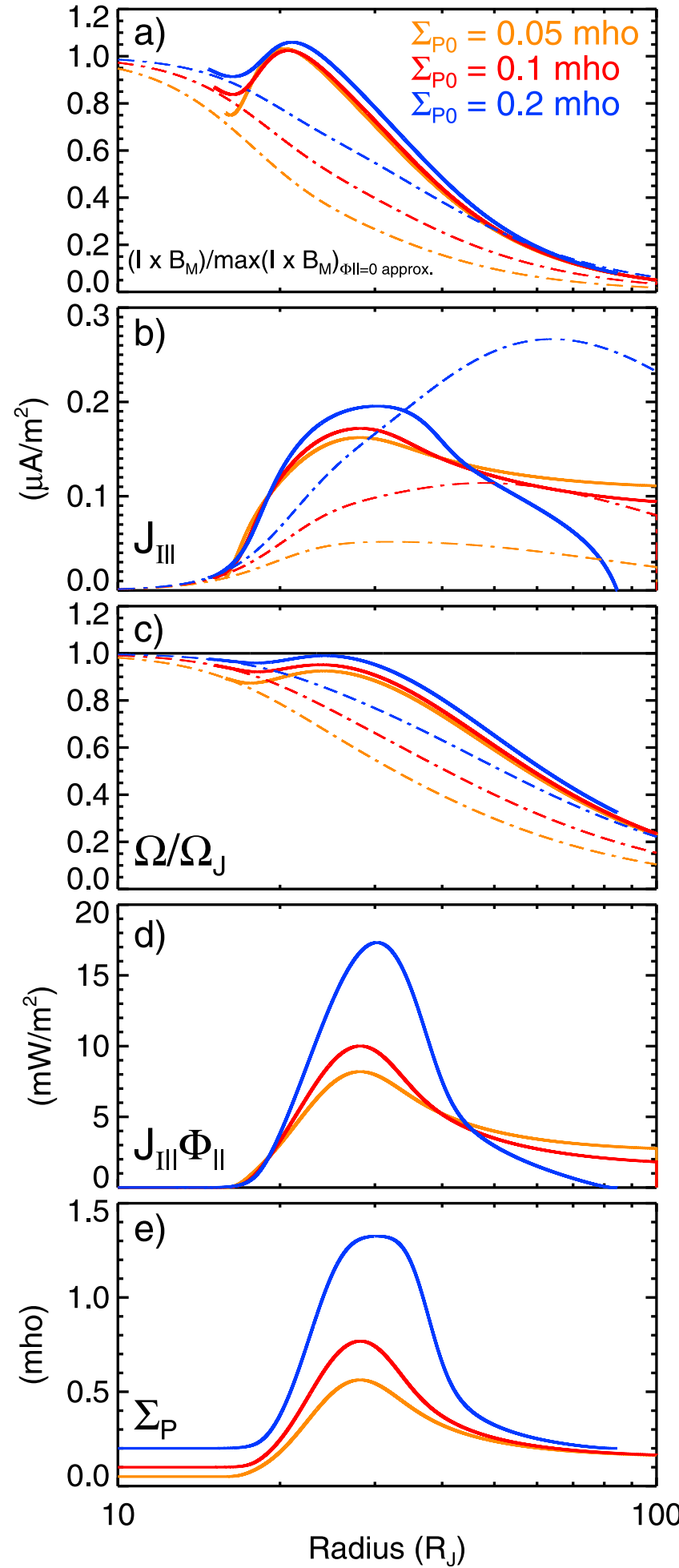

Figure 8. (a-e) Solutions for varying background Pedersen conductance of $\Sigma_{P_{0}}=0.05 \mathrm{mho}, 0.1 \mathrm{mho}$, and $0.2 \mathrm{mho}$ for an outer constraint of $\mathrm{I}_{100}=86 \mathrm{MA}$. Format is similar to Figure 4 except that Figure 8e displays the height-integrated Pedersen conductivity.

Table 2. Variation in Modeled Auroral Parameters With Auroral Cavity Electron Density for $\Phi_{\|_{\text {Max }}}=75 \mathrm{kV}, \dot{M}=1000 \mathrm{~kg} / \mathrm{s}, \Sigma_{P_{0}}=$ $0.1 \mathrm{mho}, \epsilon=1.0, \mathrm{~T}_{x}=2.5 \mathrm{keV}, R_{x}=16$

\begin{tabular}{lccccc}
\hline $\begin{array}{c}\mathrm{n}_{x} \\
\left(\mathrm{~cm}^{-3}\right)\end{array}$ & $\begin{array}{c}\mathrm{EF} \\
\left(\mathrm{mW} / \mathrm{m}^{2}\right)\end{array}$ & $\begin{array}{c}\text { Width } \\
(\mathrm{deg})\end{array}$ & $\begin{array}{c}\text { Equatorial } \\
\text { Location } \\
\left(\mathrm{R}_{J}\right)\end{array}$ & $\begin{array}{c}\mathrm{I}_{100} \\
(\mathrm{MA})\end{array}$ & $\begin{array}{c}\Omega / \Omega_{i} \\
(\%)\end{array}$ \\
\hline $0.007^{\mathrm{a}}$ & 9.8 & 0.7 & 26.5 & 66.7 & 97.8 \\
0.01 & 14.0 & 0.7 & 29.5 & 92.1 & 97.2 \\
0.03 & 42.1 & 0.7 & 43.1 & 220.3 & 95.0 \\
0.05 & 70.2 & 0.7 & 51.0 & 319.2 & 94.4 \\
\hline
\end{tabular}

${ }^{\text {a }}$ Energy flux, field-aligned potentials, and percent corotation are the maximum values after $\mathrm{Phi}$ is included in the solution.

sense of how the auroral width would change with input parameters.

[28] Ray et al. [2010] explore the variations in the solutions with the location of the acceleration region, $R_{x}$, and the efficiency of the Pedersen conductance enhancement, $\epsilon$, and this discussion occasionally refers to these studies.

\subsection{Variations With the High-Latitude Electron Density}

[29] Figure 4 displays the solutions with variation in auroral cavity electron density for $n_{x}=0.007 \mathrm{~cm}^{-3}, 0.01 \mathrm{~cm}^{-3}$, $0.03 \mathrm{~cm}^{-3}$, and $0.05 \mathrm{~cm}^{-3}$. The high-latitude electron density is assumed to be that of the hot electron population. The boundary condition $\left(\frac{d \Phi_{\|}}{d r}\right)_{0}$ is directly related to $n_{x}$, with the smallest, $\left(\frac{d \Phi_{\|}}{d r}\right)_{0}=1.6 \times 10^{-5}$, for the case where $n_{x}=0.007 \mathrm{~cm}^{-3}$ and the largest, $\left(\frac{d \Phi_{\|}}{d r}\right)_{0}=9.8 \times 10^{-5}$, for $n_{x}=0.05 \mathrm{~cm}^{-3}$. The solutions for the $n_{x}=0.007 \mathrm{~cm}^{-3}$ and $0.01 \mathrm{~cm}^{-3}$ cases follow the result presented in section 3 . The solutions for the higher density cases, $n_{x}=0.03 \mathrm{~cm}^{-3}$ and $0.05 \mathrm{~cm}^{-3}$ present slightly different trends. The key auroral parameters are summarized in Table 2 for each case.

[30] The electron thermal current density, $J_{\text {crit }}$, is directly proportional to the high-latitude electron density. Therefore, changes in $n_{x}$ significantly affect the solution by shifting $r_{c r i t}$, the location at which the field-aligned current density is greater than the critical current density and field-aligned potentials develop. The critical radius moves to larger equatorial distances with increasing $n_{x}$. We choose an outer constraint of $\Phi_{\|_{M a x}}=75 \mathrm{kV}$ for these solutions as no solutions exist for an $\mathrm{I}_{100}$ as low as $86 \mathrm{MA}$ for large densities $\left(n_{x}>\right.$ $0.02 \mathrm{~cm}^{-3}$ ) and $\dot{M}=1000 \mathrm{~kg} / \mathrm{s}, R_{x}=16, \Sigma_{P_{0}}=0.1 \mathrm{mho}$, $T_{x}=2.5 \mathrm{keV}$.

Table 3. Variation in Modeled Auroral Parameters With Electron Temperature for $\mathrm{I}_{100}=86 \mathrm{MA}, \dot{M}=1000 \mathrm{~kg} / \mathrm{s}, \Sigma_{P_{0}}=0.1 \mathrm{mho}, \epsilon=$ $1.0, n_{x}=0.01 \mathrm{~cm}^{-3}, R_{x}=16^{\mathrm{a}}$

\begin{tabular}{cccccc}
\hline $\begin{array}{c}\mathrm{T}_{x} \\
(\mathrm{keV})\end{array}$ & $\begin{array}{c}\mathrm{EF} \\
\left(\mathrm{mW} / \mathrm{m}^{2}\right)\end{array}$ & $\begin{array}{c}\text { Width } \\
(\mathrm{deg})\end{array}$ & $\begin{array}{c}\text { Equatorial } \\
\text { Location } \\
\left(\mathrm{R}_{J}\right)\end{array}$ & $\begin{array}{c}\Phi_{\|} \\
(\mathrm{kV})\end{array}$ & $\begin{array}{c}\Omega / \Omega_{J} \\
(\%)\end{array}$ \\
\hline 1.0 & 37.2 & 0.8 & 34.6 & 274.9 & 105.9 \\
1.5 & 11.6 & 0.8 & 28.6 & 72.8 & 97.0 \\
2.5 & 10.0 & 0.7 & 28.2 & 58.2 & 95.1 \\
5.0 & 11.7 & 0.7 & 28.4 & 64.2 & 96.1 \\
\hline
\end{tabular}

${ }^{\mathrm{a}}$ Energy flux, field-aligned potentials, and percent corotation are the maximum values after Phi is included in the solution. 
Table 4. Variation in Modeled Auroral Parameters With Radial Mass Transport Rate for $\mathrm{I}_{100}=86 \mathrm{MA}, \Sigma_{P_{0}}=0.1 \mathrm{mho}, \epsilon=1.0$, $T_{x}=2.5 \mathrm{keV}, n_{x}=0.01 \mathrm{~cm}^{-3}, R_{x}=16^{\mathrm{a}}$

\begin{tabular}{lccccc}
\hline$\dot{M}$ & $\begin{array}{c}\mathrm{EF} \\
(\mathrm{kg} / \mathrm{s})\end{array}$ & $\begin{array}{c}\text { Width } \\
\left(\mathrm{mW} / \mathrm{m}^{2}\right)\end{array}$ & $\begin{array}{c}\text { Equatorial } \\
\text { Location } \\
\left(\mathrm{R}_{J}\right)\end{array}$ & $\begin{array}{c}\Phi_{\|} \\
(\mathrm{kV})\end{array}$ & $\begin{array}{c}\Omega / \Omega_{J} \\
(\%)\end{array}$ \\
\hline 500 & 24.8 & 0.6 & 41.2 & 120.3 & 100.6 \\
1000 & 10.0 & 0.7 & 28.2 & 58.2 & 95.1 \\
2000 & 5.2 & 1.1 & 22.8 & 37.0 & 93.6 \\
5000 & 2.5 & 1.6 & 19.9 & 23.5 & 91.3 \\
\hline
\end{tabular}

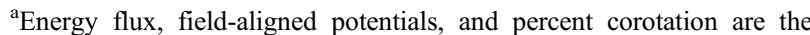
maximum values after Phi is included in the solution.

[31] The maximum $J_{\|}^{I}$ increases with $n_{x}$, reflecting the proportionality of $J_{\text {crit }}$ to $n_{x}$. As $r_{\text {crit }}$ increases with $n_{x}, J_{\|}^{I}$ peaks at $\sim 40 \mathrm{R}_{J}$ and $\sim 50 \mathrm{R}_{J}$ for $n_{x}=0.03 \mathrm{~cm}^{-3}$ and $0.05 \mathrm{~cm}^{-3}$, respectively. At such large equatorial distances the magnetic field strength is weak and hence the maximum $\mathbf{I} \times \mathbf{B}$ force occurs $\sim 10 \mathrm{R}_{J}$ before the peak in $J_{\|}^{I}$ where the magnetic field strength is stronger. The angular velocity profile follows from the $\mathbf{I} \times \mathbf{B}$ profiles with the plasma remaining near rigid corotation past $30 \mathrm{R}_{J}$.

[32] The energy flux profile peaks where $J_{\|}^{I}$ is a maximum as it is directly proportional to both $\Phi_{\|}$and $J_{\|}^{I}$. The maximum energy flux increases with $n_{x}$ as larger currents exist in the system and we hold $\Phi_{\|_{\max }}$ fixed. The auroral emission maps to an equatorial distance of $\sim 51 \mathrm{R}_{J}$ for $n_{x}=0.05 \mathrm{~cm}^{-3}$, outside the $20-30 \mathrm{R}_{J}$ predicted by auroral observations, and has a narrow width of $\sim 0.7^{\circ}$ at the atmosphere. For $n_{x}=$ $0.03 \mathrm{~cm}^{-3}$, the auroral emission maps to $\sim 43 \mathrm{R}_{J}$ and also has a width of $\sim 0.7^{\circ}$ as the stretched magnetic field outside of $\sim 20 \mathrm{R}_{J}$ concentrates the middle magnetosphere onto a narrow latitudinal region at the planet. The peak energy fluxes of $\sim 42 \mathrm{~mW} / \mathrm{m}^{2}$ and $\sim 70 \mathrm{~mW} / \mathrm{m}^{2}$ are larger than the 2-30 $\mathrm{mW} / \mathrm{m}^{2}$ derived by Gustin et al. [2004]; however, they are consistent with energy fluxes derived from observations of bright dawn auroral arcs [Gustin et al., 2006]. The radial currents for the $n_{x}=0.03 \mathrm{~cm}^{-3}$ and $0.05 \mathrm{~cm}^{-3}$ cases are two to three times larger than those measured by Galileo [Khurana, 2001].

[33] It is unlikely that the high-latitude electron density is much larger than $0.01 \mathrm{~cm}^{-3}$ as for large densities, e.g. $n_{x} \sim 0.1 \mathrm{~cm}^{-3}$, the thermal current density is such that the criteria for the development of field-aligned potentials, $J_{\|}^{I}>J_{\text {crit }}$, is never met. However, due to the presence of bright aurora this cannot be the case. Additionally, the high densities investigated here, $n_{x}=0.3 \mathrm{~cm}^{-3}$ and $n_{x}=0.5 \mathrm{~cm}^{-3}$, result in unrealistic radial currents and

Table 5. Variation in Modeled Auroral Parameters With Radial Mass Transport Rate for $\Phi_{\|_{\text {Max }}}=75 \mathrm{kV}, \Sigma_{P_{0}}=0.1 \mathrm{mho}, \epsilon=1.0$, $T_{x}=2.5 \mathrm{keV}, n_{x}=0.01 \mathrm{~cm}^{-3}, R_{x}=16^{\mathrm{a}}$

\begin{tabular}{lccccc}
\hline $\begin{array}{c}\dot{M} \\
(\mathrm{~kg} / \mathrm{s})\end{array}$ & $\begin{array}{c}\mathrm{EF} \\
\left(\mathrm{mW} / \mathrm{m}^{2}\right)\end{array}$ & $\begin{array}{c}\text { Width } \\
(\mathrm{deg})\end{array}$ & $\begin{array}{c}\text { Equatorial } \\
\text { Location } \\
\left(\mathrm{R}_{J}\right)\end{array}$ & $\begin{array}{c}I_{100} \\
(\mathrm{MA})\end{array}$ & $\begin{array}{c}\Omega / \Phi_{J} \\
(\%)\end{array}$ \\
\hline 500 & 14.0 & 0.7 & 36.8 & 77.8 & 97.6 \\
1000 & 14.0 & 0.7 & 29.5 & 92.1 & 97.2 \\
2000 & 14.0 & 0.8 & 24.7 & 108.8 & 96.3 \\
5000 & 14.0 & 1.0 & 20.7 & 139.0 & 94.2 \\
\hline
\end{tabular}

${ }^{\mathrm{a}}$ Energy flux, field-aligned potentials, and percent corotation are the maximum values after Phi is included in the solution.
Table 6. Variation in Modeled Auroral Parameters With Background Pedersen Conductance for $\mathrm{I}_{100}=86 \mathrm{MA}, \dot{M}=1000 \mathrm{~kg} / \mathrm{s}$, $\Sigma_{P_{0}}=0.1 \mathrm{mho}, \epsilon=1.0, n_{x}=0.01 \mathrm{~cm}^{-3}, R_{x}=16^{\mathrm{a}}$

\begin{tabular}{lccccc}
\hline $\begin{array}{c}\Sigma_{P_{0}} \\
(\mathrm{mho})\end{array}$ & $\begin{array}{c}\mathrm{EF} \\
\left(\mathrm{mW} / \mathrm{m}^{2}\right)\end{array}$ & $\begin{array}{c}\text { Width } \\
(\mathrm{deg})\end{array}$ & $\begin{array}{c}\text { Equatorial } \\
\text { Location } \\
\left(\mathrm{R}_{J}\right)\end{array}$ & $\begin{array}{c}\Phi_{\|} \\
(\mathrm{kV})\end{array}$ & $\begin{array}{c}\Omega / \Omega_{J} \\
(\%)\end{array}$ \\
\hline 0.05 & 8.2 & 1.0 & 28.2 & 50.5 & 92.5 \\
0.1 & 10.0 & 0.7 & 28.2 & 58.2 & 95.1 \\
0.2 & 17.3 & 1.4 & 30.3 & 88.6 & 99.0 \\
\hline
\end{tabular}

${ }^{\text {a}}$ Energy flux, field-aligned potentials, and percent corotation are the maximum values after Phi is included in the solution.

magnetospheric angular velocity profiles that do not deviate significantly from corotation until $\sim 40-50 \mathrm{R}_{J}$ which is inconsistent with in situ measurements of the magnetospheric plasma. Alternately, it is unlikely that the high-latitude electron density is less than $0.005 \mathrm{~cm}^{-3}$ as $r_{\text {crit }}$ moves in toward Jupiter resulting in strong currents that drive the plasma to super-corotation in order to match the radial currents observed by Galileo [Khurana, 2001] and satisfy the outer constraint of $\mathrm{I}_{100}=86 \mathrm{MA}$.

\subsection{Variations With the Electron Temperature}

[34] Figure 5 shows the variation in solutions with the temperature of the high-latitude electron population, $T_{x}=$ $1.0 \mathrm{keV}, 1.5 \mathrm{keV}, 2.5 \mathrm{keV}$ and $5.0 \mathrm{keV}$, and an outer constraint of $\mathrm{I}_{100}=86 \mathrm{MA}$. The boundary condition $\left(\frac{d \Phi_{\|}}{d r}\right)_{0}$ is directly related to $T_{x}$, with the smallest, $\left(\frac{d \Phi_{\|}}{d r}\right)_{0}=4.0 \times 10^{-6}$, for the case where $T_{x}=1.0 \mathrm{keV}$ and the largest, $\left(\frac{d \Phi_{\|}}{d r}\right)_{0}=$ $6.7 \times 10^{-5}$, for $T_{x}=5.0 \mathrm{keV}$. The solutions for $T_{x}=1.5 \mathrm{keV}$, $2.5 \mathrm{keV}$, and $5.0 \mathrm{keV}$ follow those presented in section 3 while the solution for $T_{x}=1.0 \mathrm{keV}$ is similar to that presented in section 4.5 of Ray et al. [2010] for $R_{x}=11$. The key auroral parameters are summarized in Table 3 for each case.

[35] The electron thermal current density, $J_{c r i t}$, is directly proportional to the square root of the electron temperature. Therefore, increases in $T_{x}$ move $r_{c r i t}$ out from Jupiter, but not to the extent shown in section 4.1 . For $T_{x}=1.0 \mathrm{keV}$, $J_{\|}^{I}$ grows steeply over a narrow radial range before flattening at $\sim 20 \mathrm{R}_{J}$ due to the saturation of the field-aligned current density, and finally declining again at $\sim 50 \mathrm{R}_{J}$. As $r_{\text {crit }}$ is closer to Jupiter for the $T_{x}=1 \mathrm{keV}$ case than for the other cases, the steep initial growth of $J_{\|}^{I}$ with radial distance occurs where the equatorial magnetic field is stronger. Hence the corresponding $\mathbf{I} \times \mathbf{B}$ force is greater than for cases with $T_{x}>1.0 \mathrm{keV}$. In the region from $\sim 21-50 \mathrm{R}_{J}$, the fieldaligned current density is saturated as the entire electron distribution has been moved into the loss cone. The fieldaligned potential continues to increase with the energy of the precipitating electrons exceeding $80 \mathrm{keV}$ such that the modified Pedersen conductance profile has a double peaked structure [Ray et al., 2010]. The field-aligned potential and incident energy flux profiles turn over at $\sim 35 \mathrm{R}_{J}$, but the field-aligned current density does not decrease immediately with the change in $d \Phi_{\|} / d r$ due to the saturation of $J_{\|}^{I}$.

[36] The angular velocity profiles are nearly identical for $T_{x}=1.5 \mathrm{keV}, 2.5 \mathrm{keV}$, and $5.0 \mathrm{keV}$ with the equatorial 
plasma remaining near rigid corotation out to $\sim 35 \mathrm{R}_{J}$. The large $\mathbf{I} \times \mathbf{B}$ force for the $T_{x}=1.0 \mathrm{keV}$ case accelerates the magnetospheric plasma to super-corotational velocities in the middle magnetosphere and, therefore, is not a physical solution.

[37] The auroral emission width and brightness vary greatly between the case of $T_{x}=1.0 \mathrm{keV}$ and the cases of $T_{x}=1.5 \mathrm{keV}, 2.5 \mathrm{keV}$, and $5.0 \mathrm{keV}$. For the latter three cases, the width of the auroral emission is nearly constant at $\sim 0.7^{\circ}$ at Jupiter's atmosphere and maps to the same equatorial location of $\sim 28 \mathrm{R}_{J}$. For $T_{x}=1.0 \mathrm{keV}$, the energy flux incident on the ionosphere is over three times greater than in the other cases, peaking at $\sim 38 \mathrm{~mW} / \mathrm{m}^{2}$. The intense aurora is due to the low critical current density and resulting saturation of $J_{\|}^{I}$. When $J_{\|}^{I}$ is saturated $\Phi_{\|}$increases until the magnetospheric plasma lag, primarily due to the decrease in the equatorial $\mathbf{I} \times \mathbf{B}$ force associated with the diminishing equatorial magnetic field strength with radius, is such that the magnitude of $E_{M}$ surpasses that of $E_{I}$. The incident energy flux increases with $\Phi_{\|}$and the auroral oval slightly broadens, mapping to an ionospheric width of $\sim 0.8^{\circ}$. For $T_{x}=1.0 \mathrm{keV}$ the auroral emission maps to an equatorial radius of $\sim 35 \mathrm{R}_{J}$. The Pedersen conductance feedback, which is related to the energy flux incident on the ionosphere and the electron precipitation energy, peaks at roughly the same value for the cases of $T_{x}=1.5 \mathrm{keV}, 2.5 \mathrm{keV}$, and $5.0 \mathrm{keV}$.

\subsection{Variations With the Mass Transport Rate}

[38] Figures 6 and 7 display the variation in solutions with radial mass transport rates of $\dot{M}=500 \mathrm{~kg} / \mathrm{s}, 1000 \mathrm{~kg} / \mathrm{s}$, $2000 \mathrm{~kg} / \mathrm{s}$ and $5000 \mathrm{~kg} / \mathrm{s}$, and outer constraints of $\mathrm{I}_{100}=$ $86 \mathrm{MA}$ and $\Phi_{\|_{\text {Max }}}=75 \mathrm{kV}$, respectively. For both outer constraints, $\mathrm{I}_{100} \stackrel{{ }_{\text {Max }}}{=} 86 \mathrm{MA}$ and $\Phi_{\|_{M a x}}=75 \mathrm{kV}$, the boundary condition $\left(\frac{d \Phi_{\|}}{d r}\right)_{0}$ is directly related to $\dot{M}$, with the smallest $\left(\frac{d \Phi_{\|}}{d r}\right)_{0}$ for the case where $\dot{M}=500 \mathrm{~kg} / \mathrm{s},\left(\frac{d \Phi_{\|}}{d r}\right)_{0}=9.6 \times 10^{-6}$ and $9.3 \times 10^{-6}$, respectively, and the largest for $\dot{M}=$ $5000 \mathrm{~kg} / \mathrm{s},\left(\frac{d \Phi_{\|}}{d r}\right)_{0}=1.4 \times 10^{-5}$ and $2.8 \times 10^{-5}$, respectively. The key auroral parameters are summarized in Tables 4 and 5 for each case.

[39] Unlike the parameters explored previously $\left(\epsilon, R_{x}, n_{x}\right.$, $T_{x}$ ), the radial mass transport rate affects the solutions through the $\Phi_{\|}=0$ approximation rather than through the current-voltage relation or the modification of the Pedersen conductance. As $\dot{M}$ increases, the equatorial plasma puts a larger stress on the system with the plasma angular velocity departing from rigid corotation at smaller equatorial distances. The equatorial plasma demands more angular momentum from Jupiter and therefore $J_{\|}^{I}$ exceeds $J_{c r i t}$ closer to Jupiter for larger $\dot{M}$. At radial distances larger than $r_{c r i t}$, the solution behavior depends on the outer constraint. For an outer constraint of $\mathrm{I}_{100}=86 \mathrm{MA}$, the field-aligned current profiles are counterintuitive, with the largest $J_{\|}^{I}$ occurring for the smallest $\dot{M}$. As $\dot{M}$ increases, there is a greater demand for angular momentum at smaller equatorial distances and hence increased field-aligned currents. Subsequently, this larger load results in an increased radial current closer to Jupiter such that less current is demanded farther out in the system to satisfy the outer constraint. For an outer constraint of $\Phi_{\|_{M a x}}=75 \mathrm{kV}$, the maximum field-aligned current is the same for all $\dot{M}$ due to the current-voltage relation. The fieldaligned current density profile peaks at smaller equatorial distances for larger $\dot{M}$ as expected due to the large stresses on the system for high radial mass transport rates.

[40] For both outer constraints, the increased $\mathbf{I} \times \mathbf{B}$ force peaks at smaller equatorial distances for larger $\dot{M}$. However, for an outer constraint of $\mathrm{I}_{100}=86 \mathrm{MA}$, the $\mathbf{I} \times \mathbf{B}$ force does not surpass the initial I $\times \mathbf{B}$ force for $\dot{M}=2000 \mathrm{~kg} / \mathrm{s}$ and $5000 \mathrm{~kg} / \mathrm{s}$ and as such, the plasma does not remain near rigid corotation. For $\dot{M}=5000 \mathrm{~kg} / \mathrm{s}$, the angular velocity profile is near that of the $\Phi_{\|}=0$ approximation with the plasma gaining only a slight push toward corotation from the $\mathbf{I} \times \mathbf{B}$ force. For $\dot{M}=500 \mathrm{~kg} / \mathrm{s}$, the plasma remains near rigid corotation, with a maximum angular velocity of roughly corotation at $\sim 30 \mathrm{R}_{J}$.

[41] When an outer constraint of $\Phi_{\|_{\text {Max }}}=75 \mathrm{kV}$ is used, the maximum $\mathbf{I} \times \mathbf{B}$ force surpasses the initial $\mathbf{I} \times \mathbf{B}$ force for all $\dot{M}$. The plasma remains near rigid corotation for all cases. However, the return toward corotation is strongest for the $\dot{M}=5000 \mathrm{~kg} / \mathrm{s}$ case as the initial departure from corotation is the largest.

[42] For the outer constraint of $\mathrm{I}_{100}=86 \mathrm{MA}$, the auroral emission is $\operatorname{dim}$ for $\dot{M}=2000 \mathrm{~kg} / \mathrm{s}$ and $5000 \mathrm{~kg} / \mathrm{s}$ with peak energy fluxes of $\sim 5 \mathrm{~mW} / \mathrm{m}^{2}$ and $\sim 2 \mathrm{~mW} / \mathrm{m}^{2}$ respectively. The width of the emission for $\dot{M}=2000 \mathrm{~kg} / \mathrm{s}$ is $\sim 1.1^{\circ}$ at the atmosphere and maps to $\sim 23 \mathrm{R}_{J}$, while the emission for $\dot{M}=$ $5000 \mathrm{~kg} / \mathrm{s}$ is broader at $\sim 1.6^{\circ}$ and maps to $\sim 20 \mathrm{R}_{J}$. The auroral emission for $\dot{M}=500 \mathrm{~kg} / \mathrm{s}$ is the brightest and narrowest with an incident energy flux of $\sim 25 \mathrm{~mW} / \mathrm{m}^{2}$ and atmospheric width of $\sim 0.6^{\circ}$. However, the emission maps to an equatorial radius of $\sim 41 \mathrm{R}_{J}$, outside the range predicted by observations. Counter intuitively a smaller radial mass transport rate drives a brighter auroral emission. This result is a direct consequence of the outer constraint, $\mathrm{I}_{100}=86 \mathrm{MA}$. A larger magnetospheric load demands more current at smaller equatorial radial distances. This provides a large contribution to the total radial current and reduces the fieldaligned current density mapping to middle and outer magnetosphere. Subsequently, the modeled auroral emission is dimmer for a large $\dot{M}$.

[43] The solutions for $\Phi_{\|_{M a x}}=75 \mathrm{kV}$ make more physical sense than those for $\mathrm{I}_{100}=86 \mathrm{MA}$. The auroral oval maps to smaller equatorial distances for large $\dot{M}$. Larger radial mass transport rates stress the magnetic field to a greater degree. Rigid corotation breaks down closer to Jupiter and a larger transfer of angular momentum, hence larger currents, is necessary to keep the plasma near corotation. As the maximum field-aligned potential is fixed to $75 \mathrm{kV}$, the peak brightness of the auroral emission is the same for all cases since the parameters for the current-voltage relation are independent of the radial mass transport rate. The total radial current increases with the radial mass transport rate.

\subsection{Variations With the Background Pedersen Conductance}

[44] Figure 8 displays the variation in solutions with background Pedersen conductance, $\Sigma_{P_{0}}=0.05 \mathrm{mho}, 0.1$ mho and $0.2 \mathrm{mho}$, and an outer constraint of $\mathrm{I}_{100}=86 \mathrm{MA}$. 
The boundary condition $\left(\frac{d \Phi_{\|}}{d r}\right)_{0}$ is inversely related to $\Sigma_{P_{0}}$, with $\left(\frac{d \Phi_{\|}}{d r}\right)_{0}=7.6 \times 10^{-6}$ for the case where $\Sigma_{P_{0}}=0.2 \mathrm{mho}$ and $\left(\frac{d \Phi_{\|}}{d r}\right)_{0}=7.1 \times 10^{-5}$ when $\Sigma_{P_{0}}=0.05$ mho. The solutions for $\Sigma_{P_{0}}=0.05$ mho and 0.1 mho follow those presented in section 3 while the solution for $\Sigma_{P_{0}}=0.2$ mho is markedly different. The key auroral parameters are summarized in Table 6 for each case.

[45] The background Pedersen conductance affects the solutions through the $\Phi_{\|}=0$ approximation, similar to the radial mass transport rate case. A small Pedersen conductance, $\Sigma_{P_{0}}=0.05$ mho, limits the ionospheric Pedersen current and restricts the transfer of angular momentum from Jupiter to its equatorial plasma. The equatorial plasma departs from rigid corotation at small equatorial distances and the location of $r_{\text {crit }}$ moves out slightly from Jupiter, relative to the case with $\Sigma_{P_{0}}=0.1 \mathrm{mho}$, as $J_{\|}^{I}$ grows more slowly with radial distance. In the limiting case of a small $\Sigma_{P_{0}}$ (e.g. $\Sigma_{P_{0}}=0.01 \mathrm{mho}$ ), the field-aligned currents never surpass $J_{\text {crit }}$ and the system follows the $\Phi_{\|}=0$ approximation for all radial distances. Conversely, high background Pedersen conductances, e.g. $\Sigma_{P_{0}}=0.2 \mathrm{mho}$, drive larger ionospheric currents and more angular momentum is transferred from Jupiter to its equatorial plasma such that the magnetospheric plasma angular velocity remains near corotation out to larger radial distances. The field-aligned currents grow more rapidly with radial distance than in the baseline case of $\Sigma_{P_{0}}=0.1 \mathrm{mho}$ and hence $J_{\|}^{I}>J_{\text {crit }}$ closer to Jupiter.

[46] The $\mathbf{I} \times \mathbf{B}$ force reaches its maximum at nearly the same location for all cases, with the peak shifting slightly to larger equatorial distances with increasing $\Sigma_{P_{0}}$. The angular velocity profile follows the same trend, with the equatorial plasma remaining near rigid corotation out to larger radii for greater $\Sigma_{P_{0}}$. It should be noted that the equatorial plasma sustains near rigid corotation for the $\Sigma_{P_{0}}=0.2$ mho case, and slightly lesser degrees of corotation for smaller $\Sigma_{P_{0}}$ due to the larger initial departures from corotation.

[47] The maximum field-aligned current density for the case of $\Sigma_{P_{0}}=0.2 \mathrm{mho}$ is larger than for the $\Sigma_{P_{0}}=0.05 \mathrm{mho}$ and 0.1 mho cases. However, unlike the other two cases, $J_{\|}^{I}$ enters a downward current region at $\sim 85 \mathrm{R}_{J}$ in order to satisfy the outer constraint of $\mathrm{I}_{100}=86 \mathrm{MA}$ due to the large field-aligned currents in the middle magnetosphere. We do not plot the solutions to the $\Sigma_{P_{0}}=0.2$ mho case outside of $85 \mathrm{R}_{J}$ as our model does not include the physics of the downward current region.

[48] The field-aligned current density increases unphysically above $J_{\text {crit }}$ in all $\Phi_{\|}=0$ approximations. However, this is most noticeable when $\Sigma_{P_{0}}=0.2$ mho outside of $\sim 32 \mathrm{R}_{J}$ where $J_{\|}^{I}$ is larger than that for the solution with field-aligned potentials. The $\Phi_{\|}=0$ approximation does not account for the lack of current carriers at high-latitudes and hence permits $J_{\|}^{I}$ to exceed $J_{\text {crit }}$ to satisfy torque balance between the ionosphere and magnetosphere.

[49] The auroral oval maps to roughly the same equatorial location of $\sim 28 \mathrm{R}_{J}$ for the $\Sigma_{P_{0}}=0.05$ mho and 0.1 mho cases with widths of $\sim 1.0^{\circ}$ and $\sim 0.7^{\circ}$, respectively. The auroral emission is slightly dimmer for $\Sigma_{P_{0}}=0.05$ with an incident energy flux of $\sim 8 \mathrm{~mW} / \mathrm{m}^{2}$ as opposed to $\sim 10 \mathrm{~mW} / \mathrm{m}^{2}$ for $\Sigma_{P_{0}}=0.1$ mho. The emission is brightest for the case of
$\Sigma_{P_{0}}=0.2$ mho with an incident energy flux of $\sim 17 \mathrm{~mW} / \mathrm{m}^{2}$ and a width of $\sim 1.4^{\circ}$ which maps to $\sim 30 \mathrm{R}_{J}$.

\section{Discussion}

[50] The above parameter space studies extend the work presented by Ray et al. [2010] to investigate how changes in the high-latitude electron density, electron temperature, radial mass loading rate, and background Pedersen conductance affect the auroral current system. The system of equations presented in section 2 are non-linear and therefore small changes to the auroral parameters can result in large changes in the properties of the auroral emission (i.e. auroral width, brightness, and mapping location).

[51] The development of the auroral current system is similar for all parameters. While there are changes in the magnitude and radial profiles of the field-aligned currents, incident energy flux at the ionosphere, and magnetospheric $\mathbf{I} \times \mathbf{B}$ force, the general current system trends remain the same. The solutions follow the $\Phi_{\|}=0$ approximation until $J_{\|}^{I}$ exceeds $J_{\text {crit }}$ at $r_{\text {crit }}$. At this location, the field-aligned currents and potentials grow, modifying the Pedersen conductance with electron precipitation and transferring angular momentum to the magnetospheric plasma. The auroral energy flux increases with $\Phi_{\|}$. As the equatorial magnetic field strength decreases with radial distance, the $\mathbf{I} \times \mathbf{B}$ force in the magnetosphere declines and the magnetospheric plasma lags corotation. The field-aligned potentials turn over and the auroral energy flux decreases, creating an auroral emission of finite width at the ionosphere. The precise behavior of the solutions varies with slight modifications to the mass loading rate, high latitude electron density and temperature, and background Pedersen conductance. Modifications in $\dot{M}$ and $\Sigma_{P_{0}}$ modify the $\Phi_{\|}=0$ approximation and therefore affect the solution for the entire spatial domain. The high-latitude electron density and temperature define the electron thermal current and therefore affect the solutions from $r=r_{\text {crit }}$ to the outer boundary at $100 \mathrm{R}_{J}$.

[52] Where $d \Phi_{\|} / d r>0$, the locations where $\frac{d\left(J_{I} \Phi_{\|}\right)}{d r}>0$ in Figures $4 \mathrm{~d}, 5 \mathrm{~d}, 6 \mathrm{~d}, 7 \mathrm{~d}$, and $8 \mathrm{~d}$, the magnitude of $E_{I}$ is greater than that of $E_{M}$, corresponding to a larger deviation from corotation in the ionosphere than in the magnetosphere. Conversely, where $d \Phi_{\|} / d r<0$ the magnitude of $E_{M}$ is greater than that of $E_{I}$ and hence the magnetospheric plasma lags corotation more than the ionospheric plasma. The local enhancement of $E_{I}^{*}$ relative to $\left|E_{M}\right|$ is consistent with the results presented by Ergun et al. [2009] for the Io flux tube and is a general property of any upward current region with parallel electric fields when $\nabla \times E=0$. We note that, globally, the net transfer of angular momentum is from the ionosphere to the magnetosphere and refer the reader to the discussion by Ergun et al. [2009] for the Io flux tube.

[53] We hold the outer constraint fixed to $\mathrm{I}_{100}=86 \mathrm{MA}$ for all studies. However the parameter space study for $n_{x}$ has an outer constraint of $\Phi_{\|_{\operatorname{Max}}}=75 \mathrm{kV}$ as solutions do not exist for all of the investigated parameters and we show the solutions for both $\mathrm{I}_{100}=86 \mathrm{MA}$ and $\Phi_{\|_{\text {Max }}}=75 \mathrm{kV}$ with variations in $\dot{M}$. The outer constraint can have a pronounced effect on the system behavior. This is most notably displayed in section 4.3 where a large $\dot{M}$ drives either the dimmest auroral emission for an outer constraint of $\mathrm{I}_{100}=86 \mathrm{MA}$ or 
the broadest emission for $\Phi_{\|_{\text {Max }}}=75 \mathrm{kV}$, and in section 4.4 where the outer constraint of $\mathrm{I}_{100}=86$ MA drives a downward current region for $\Sigma_{P_{0}}=0.2$ mho. Alternative constraints such as prescribing the radial angular velocity profile in the magnetosphere similar to Ergun et al. [2009] may allow for a more robust solution method. The variation in solutions with $\dot{M}$ and a fixed outer constraint of $\mathrm{I}_{100}=$ 86 MA suggest that the physical system does not hold a fixed outer constraint for all conditions, i.e. the radial current at the outer boundary likely varies with changes in the radial mass transport rate and local time. There is evidence for this variation in the Galileo magnetometer data [Khurana, 2001].

[54] The density or temperature of the current-carrying population are unlikely to be $n_{x}<0.005 \mathrm{~cm}^{-3}$ or $T_{x} \leq$ $1.0 \mathrm{keV}$, respectively. At such low energies and densities, the field-aligned current saturates such that the magnetosphere super-corotates and large field-aligned potentials $(\sim 275 \mathrm{kV})$ develop in order to satisfy the outer boundary condition. Gustin et al. [2006] derived precipitating electron energies up to $\sim 460 \mathrm{keV}$ from bright auroral storm emissions in the dawn main auroral oval. Additionally, the plasma in the dawn sector remains near corotation out to $\sim 40 \mathrm{R}_{J}$ with some indication of corotational flow [Krupp et al., 2001]. However, in the remainder of the magnetosphere, the magnetospheric plasma lags corotation [Krupp et al., 2001] and in the absence of dawn auroral storms, the mean energy of precipitating electrons is $\sim 30-\sim 200 \mathrm{keV}$ [Gustin et al., 2004]. Local time asymmetries in the current-carrying population could explain this variation. However at this time the magnetospheric electron population in the 1 to hundreds of $\mathrm{keV}$ range has not yet been adequately measured to determine the electron temperature variations with local time.

[55] The parameter space study for $\Sigma_{P_{0}}$ may be the most interesting. In our model, increases in the background Pedersen conductance drive a downward current region for an outer constraint of $\mathrm{I}_{100}=86 \mathrm{MA}$. This downward current region occurs in the outer magnetosphere at $\sim 80 \mathrm{R}_{J}$, but its radial location will vary with the other parameters $\left(\dot{M}, T_{x}\right.$, $\left.R_{x}, n_{x}\right)$. Khurana [2001] used Galileo data to determine that the downward current exists between 08:00 and 13:00 LT between $\sim 25$ and $50 \mathrm{R}_{J}$. Additionally, Radioti et al. [2008] observed a break in the main auroral emission in the prenoon sector and postulated that this dark region is likely connected to a downward current region.

[56] While the background Pedersen conductance at Jupiter has not yet been determined directly from atmospheric measurements, Tao et al. [2010] found that solar EUV flux produces a background Pedersen conductance of $6 \times 10^{-4}$ mho on the nightside and $6 \times 10^{-2}$ mho on the dayside of Jupiter in the absence of field-aligned currents driven by plasma inertia. Our model shows that it is unlikely that the background Pedersen conductance is as low as $6 \times 10^{-4}$ mho. At this low level, the ionospheric conductance would be the sole limiter of the currents transferring angular momentum to the magnetospheric plasma and the plasma would approach stagnation close to Jupiter, by $\sim 40 \mathrm{R}_{J}$.

\section{Conclusions}

[57] We have extended the model presented by Ray et al. [2010] to investigate how the current system associated with the radial transport of Iogenic plasma varies with system parameters. This current system drives Jupiter's main auroral emission and we have also described how the brightness and width of the main aurora is expected to vary with radial mass transport rate, background Pedersen conductance, and the temperature and density of the currentcarrying hot electron population. This is dependent on the selected outer constraint, either $\mathrm{I}_{100}$ or $\Phi_{\|_{\text {Mar }}}$, as most clearly shown in the case of varying radial mass transport rates. Therefore it is reasonable to expect that the conclusions drawn here will be modified if different outer constraints are imposed or if the outer constraint varies with local time. We draw the following conclusions using an outer constraint of $\mathrm{I}_{100}=86$ MA.

[58] 1. The upper limit for the high-latitude electron density is $\sim 0.01 \mathrm{~cm}^{-3}$. For larger electron densities, the auroral oval maps to larger magnetospheric distances than predicted by in-situ measurements and HST observations.

[59] 2. The lower limit for the high-latitude electron temperature is $\sim 1.5 \mathrm{keV}$. For electron temperatures less than $1.5 \mathrm{keV}$, the field-aligned currents saturate, resulting in the super-corotation of the magnetospheric plasma.

[60] 3. The lower limit for the background Pedersen conductance is $\sim 0.01$ mho. Below $0.01 \mathrm{mho}$, the ionosphere cannot conduct the necessary currents to keep the magnetospheric plasma near corotation as observed.

[61] 4. The auroral emission broadens with increases in the radial mass transport rate.

[62] The Juno mission, which is due to arrive at Jupiter in 2016, will make measurements of Jupiter's auroral region including the density and temperature of the high-latitude electron population which will further our understanding of the auroral current system.

[63] Acknowledgments. L. C. R. was supported for this work by NASA's NESSF program.

[64] Masaki Fujimoto thanks the reviewers for their assistance in evaluating this paper.

\section{References}

Clarke, J. T., D. Grodent, S. W. H. Cowley, E. J. Bunce, P. Zarka, J. E. P. Connerney, and T. Satoh (2004), Jupiter's aurora, in Jupiter: The Planet, Satellites and Magnetosphere, pp. 639-670, Cambridge Univ. Press, Cambridge, U. K.

Cowley, S. W. H., and E. J. Bunce (2001), Origin of the main auroral oval in Jupiter's coupled magnetosphere-ionosphere system, Planet. Space Sci., 49, 1067-1088.

Cowley, S. W. H., E. J. Bunce, T. S. Stallard, and S. Miller (2003a), Jupiter's polar ionospheric flows: Theoretical interpretation, Geophys. Res. Lett., 30(5), 1220, doi:10.1029/2002GL016030.

Cowley, S. W. H., E. J. Bunce, and J. D. Nichols (2003b), Origins of Jupiter's main oval auroral emissions, J. Geophys. Res., 108(A4), 8002, doi:10.1029/ 2002JA009329.

Delamere, P. A., and F. Bagenal (2010), Solar wind interaction with Jupiter's magnetosphere, J. Geophys. Res., 115, A10201, doi:10.1029/ 2010JA015347.

Delamere, P. A., F. Bagenal, and A. Steffl (2005), Radial variations in the Io plasma torus during the Cassini era, J. Geophys. Res., 110, A12223, doi:10.1029/2005JA011251.

Ergun, R. E., L. Ray, P. A. Delamere, F. Bagenal, V. Dols, and Y. Su (2009), Generation of parallel electric fields in the Jupiter-Io torus wake region, J. Geophys. Res., 114, A05201, doi:10.1029/2008JA013968.

Frank, L. A., and W. R. Paterson (2002), Galileo observations of electron beams and thermal ions in Jupiter's magnetosphere and their relationship to the auroras, J. Geophys. Res., 107(A12), 1478, doi:10.1029/ 2001JA009150.

Gustin, J., J.-C. Gérard, D. Grodent, S. W. H. Cowley, J. T. Clarke, and A. Grard (2004), Energy-flux relationship in the FUV Jovian aurora deduced from HST-STIS spectral observations, J. Geophys. Res., 109, A10205, doi:10.1029/2003JA010365. 
Gustin, J., S. W. H. Cowley, J.-C. Gérard, G. R. Gladstone, D. Grodent, and J. T. Clarke (2006), Characteristics of Jovian morning bright FUV aurora from Hubble Space Telescope/Space Telescope Imaging Spectrograph imaging and spectral observations, J. Geophys. Res., 111, A09220, doi:10.1029/2006JA011730.

Hill, T. W. (1979), Inertial limit on corotation, J. Geophys. Res., 84, 6554-6558.

Huang, T. S., and T. W. Hill (1989), Corotation lag of the Jovian atmosphere, ionosphere, and magnetosphere, J. Geophys. Res., 94, 3761-3765.

Khurana, K. K. (2001), Influence of solar wind on Jupiter's magnetosphere deduced from currents in the equatorial plane, J. Geophys. Res., 106, 25,999-26,016, doi:10.1029/2000JA000352.

Knight, S. (1973), Parallel electric fields, Planet. Space Sci., 21, 741-750.

Krupp, N., A. Lagg, S. Livi, B. Wilken, J. Woch, E. C. Roelof, and D. J. Williams (2001), Global flows of energetic ions in Jupiter's equatorial plane: First-order approximation, J. Geophys. Res., 106, 26,017-26,032, doi:10.1029/2000JA900138.

Krupp, N., et al. (2004), Dynamics of the Jovian magnetosphere, in Jupiter The Planet, Satellites and Magnetosphere, pp. 617-638, Cambridge Univ. Press, Cambridge, U. K.

Lyons, L. R. (1980), Generation of large-scale regions of auroral currents, electric potentials, and precipitation by the divergence of the convection electric field, J. Geophys. Res., 85, 17-24.

Mauk, B. H., B. J. Anderson, and R. M. Thorne (2002), Magnetosphereionosphere coupling at Earth, Jupiter, and beyond, in Atmospheres in the Solar System: Comparative Aeronomy, Geophys. Monogr. Ser., vol. 130, pp. 97-114, AGU, Washington, D. C.

McNutt, R. L., Jr., J. W. Belcher, J. D. Sullivan, F. Bagenal, and H. S. Bridge (1979), Departure from rigid co-rotation of plasma in Jupiter's dayside magnetosphere, Nature, 280, 803.

Millward, G., S. Miller, T. Stallard, A. D. Aylward, and N. Achilleos (2002), On the dynamics of the Jovian ionosphere and thermosphere III The modelling of auroral conductivity, Icarus, 160, 95-107, doi:10.1006/ icar.2002.6951.

Nichols, J., and S. Cowley (2004), Magnetosphere-ionosphere coupling currents in Jupiter's middle magnetosphere: Effect of precipitationinduced enhancement of the ionospheric Pedersen conductivity, Ann. Geophys., 22, 1799-1827.
Nichols, J. D., and S. W. H. Cowley (2005), Magnetosphereionosphere coupling currents in Jupiter's middle magnetosphere: Effect of magnetosphere-ionosphere decoupling by field-aligned auroral voltages, Ann. Geophys., 23, 799-808.

Pontius, D. H. (1997), Radial mass transport and rotational dynamics, J. Geophys. Res., 102, 7137-7150, doi:10.1029/97JA00289.

Pontius, D. H., Jr., and T. W. Hill (1982), Departure from corotation of the Io plasma torus: Local plasma production, Geophys. Res. Lett., 9, $1321-1324$

Radioti, A., J. Gérard, D. Grodent, B. Bonfond, N. Krupp, and J. Woch (2008), Discontinuity in Jupiter's main auroral oval, J. Geophys. Res. 113, A01215, doi:10.1029/2007JA012610.

Ray, L. C., Y. Su, R. E. Ergun, P. A. Delamere, and F. Bagenal (2009), Current-voltage relation of a centrifugally confined plasma, J. Geophys. Res., 114, A04214, doi:10.1029/2008JA013969.

Ray, L. C., R. E. Ergun, P. A. Delamere, and F. Bagenal (2010), Magnetosphere-ionosphere coupling at Jupiter: Effect of field-aligned potentials on angular momentum transport, J. Geophys. Res., 115, A09211, doi:10.1029/2010JA015423.

Saur, J., F. M. Neubauer, J. E. P. Connerney, P. Zarka, and M. G. Kivelson (2004), Plasma interaction of Io with its plasma torus, in Jupiter: The Planet, Satellites and Magnetosphere, pp. 537-560, Cambridge Univ. Press, Cambridge, U. K.

Scudder, J. D., E. C. Sittler, and H. S. Bridge (1981), A survey of the plasma electron environment of Jupiter: A view from Voyager, J. Geophys. Res., 86, 8157-8179.

Tao, C., H. Fujiwara, and Y. Kasaba (2010), Jovian magnetosphereionosphere current system characterized by diurnal variation of ionospheric conductance, Planet. Space Sci., 58, 351-364, doi:10.1016/j. pss.2009.10.005.

F. Bagenal, P. A. Delamere, and R. E. Ergun, Laboratory for Atmospheric and Space Physics, UCB392, University of Colorado at Boulder, Boulder, CO 80309, USA.

L. C. Ray, Space and Atmospheres Group, Department of Physics, Imperial College London, Prince Consort Road, London SW7 2AZ, UK. (1.ray@imperial.ac.uk) 